\title{
Transplacental Passage of Acetaminophen in Term Pregnancy
}

Joshua F. Nitsche, MD, PhD ${ }^{1}$ Avinash S. Patil, $\mathrm{MD}^{2} \quad$ Loralie J. Langman, $\mathrm{PhD}^{3}$ Hannah J. Penn ${ }^{4}$ Douglas Derleth, $\mathrm{MD}^{4}$ William J. Watson, $\mathrm{MD}^{5}$ Brian C. Brost, $\mathrm{MD}^{5}$

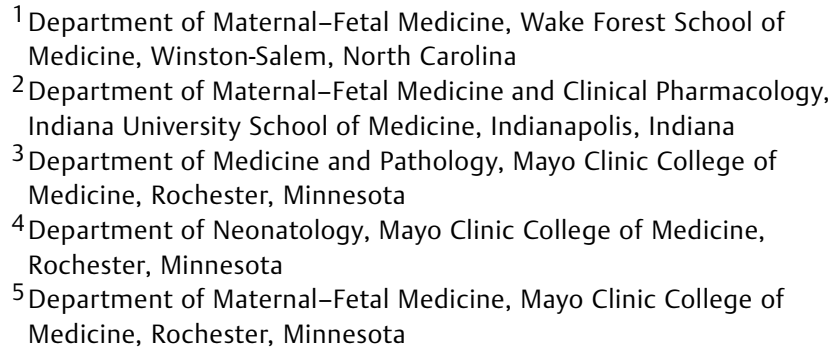

Address for correspondence Joshua F. Nitsche, MD, PhD, Department of Maternal-Fetal Medicine, Wake Forest School of Medicine, Medical Center Boulevard, Winston-Salem, NC 27157

(e-mail: jnitsche@wakehealth.edu).

Am J Perinatol 2017;34:541-543.

\begin{abstract}
Keywords

- fetal drug exposure

- acetaminophen

- pregnancy

- pharmacokinetics

Objective The objective of this study was to determine the maternal and fetal pharmacokinetic (PK) profiles of acetaminophen after administration of a therapeutic oral dose.

Study Design After obtaining Institutional Review Board approval and their written informed consent, pregnant women were given a single oral dose $(1,000 \mathrm{mg})$ of acetaminophen upon admission for scheduled cesarean delivery. Maternal venous blood and fetal cord blood were obtained at the time of delivery and acetaminophen levels were measured using gas chromatography-mass spectroscopy. PK parameters were calculated by noncompartmental analysis. Nonparametric correlation of maternal/ fetal acetaminophen levels and PK curves were calculated.

Results In this study, 34 subjects were enrolled (median, 32 years; range, 25-39 years). The median maternal weight was $82 \mathrm{~kg}$ (range, 62-100 kg). All but two subjects were delivered beyond 39 weeks' gestation. The median newborn birth weight was $3,590 \mathrm{~g}$ (interquartile range, 3,403-3,848 g). Noncompartmental analysis described similar PK parameters in the maternal ( $T_{1 / 2}, 84$ minutes; apparent clearance $[\mathrm{Cl} / \mathrm{F}], 28.8 \mathrm{~L} / \mathrm{h}$; apparent volume of distribution $\left.\left[\mathrm{V}_{\mathrm{d}} / \mathrm{F}\right], 57.5 \mathrm{~L}\right)$ and fetal compartments $\left(T_{1 / 2}, 82\right.$ minutes; $\mathrm{Cl} / \mathrm{F}, 31.2 \mathrm{~L} / \mathrm{h} ; \mathrm{V}_{\mathrm{d}} / \mathrm{F}$, $61.2 \mathrm{~L})$. Paired maternal/fetal acetaminophen levels were highly correlated $(p<0.0001)$.

Conclusion Fetal acetaminophen PKs in the fetus parallels that in the mother suggesting that placental transfer is flow limited. Maternal acetaminophen levels can be used as a surrogate for fetal exposure.
\end{abstract}

Acetaminophen is taken by nearly two-thirds of pregnant women, making it the most commonly utilized over-thecounter medication in pregnancy. ${ }^{1}$ Despite the perception of safety, maternal acetaminophen use has been associated with increased risks to the newborn of attention deficit hyperac- tivity disorder (ADHD), ${ }^{2}$ drug-induced liver injury (DILI), ${ }^{3}$ and asthma in childhood. ${ }^{4}$ The extent of fetal exposure from a standard acetaminophen dose is not well understood. Acetaminophen has been demonstrated to cross the placenta after maternal overdose ${ }^{5}$ as well as after therapeutic doses. ${ }^{6}$ Two received

June 19, 2016

accepted after revision

September 22, 2016

published online

November 2, 2016
Copyright $\odot 2017$ by Thieme Medical Publishers, Inc., 333 Seventh Avenue, New York, NY 10001, USA. Tel: +1(212) 584-4662.
DOI http://dx.doi.org/ 10.1055/s-0036-1593845. ISSN $0735-1631$. 
case reports described fetal death after maternal overdose that resulted in maternal liver injury; however, the cause of fetal death was not definitively determined in either case., Four other reports describe cases of toxic maternal levels of acetaminophen without any documented fetal sequelae. ${ }^{7-10}$ Fortunately, studies involving therapeutic doses of acetaminophen have failed to demonstrate a risk to the fetus in any trimester ${ }^{11}$ and is listed as a category B medication for use in pregnancy. In addition, a registry of more than 88,000 firsttrimester exposures to acetaminophen has not shown an increase in congenital anomalies in the fetuses exposed. ${ }^{12}$ However, the possibility of altered metabolism of the drug in the fetus raises the possibility that it could accumulate to toxic levels with chronic use.

Acetaminophen is widely used in pregnancy, ${ }^{13}$ as other pain relievers such as NSAIDs or narcotic medications carry the risk of fetal harm, namely, premature closure of the ductus arteriosus and neonatal withdrawal, respectively. ${ }^{11}$ Surprisingly, search of the PubMed database for the terms "acetaminophen," "paracetamol," and "pregnancy" revealed only one case report describing the levels of acetaminophen in the neonate after a therapeutic maternal dose of acetaminophen. ${ }^{6}$ This report suggests that the level attained in the neonate after a single maternal dose of acetaminophen is similar to the levels obtained after an oral dose administered directly to the neonate. An experimental study of normal pregnant women undergoing scheduled cesarean delivery has also shown similar acetaminophen levels in mother and neonate as soon as 30 minutes after maternal administration. ${ }^{14}$ However, the rate of decline and duration of action of acetaminophen after placental transfer has yet to be determined. In this study, we compare the maternal and fetal pharmacokinetic (PK) profiles of acetaminophen after administration of a therapeutic oral dose.

\section{Study Design}

Institutional Review Board approval was obtained for a prospective study of acetaminophen PKs in term pregnant women. After obtaining written consent, women without medical or obstetrical complications were given a single oral dose $(1,000 \mathrm{mg})$ of acetaminophen upon admission for scheduled cesarean delivery. At the time of delivery, $5 \mathrm{~mL}$ of maternal peripheral venous blood and $15 \mathrm{~mL}$ of fetal cord blood were obtained at the time of delivery. Acetaminophen and its deuterium labeled internal standard, acetaminophen $\mathrm{d}_{4}$, were purchased from Cerilliant (Round Rock, TX). All solvents were high performance liquid chromatography grade or better, and all chemicals were American Chemical Society grade. Solid phase extraction was followed by derivatization using BSTFA 1\% TMCS. One microliter of prepared sample was injected onto an Agilent gas chromatography-mass spectroscopy system (Agilent Technologies, Inc., Santa Clara, CA). Quantitation was performed using selected ion monitoring and concentrations were calculated based on a fivepoint calibration curve using a $1 / x$ linear curve fit. The calibrators ranged from 0.50 to $15 \mu \mathrm{g} / \mathrm{mL}$ and were included with each analytical run. Samples $>15 \mu \mathrm{g} / \mathrm{mL}$ were diluted with drug-free serum and reanalyzed. Each maternal/child pair contributed one set of values to the analysis. PK parameters were calculated by noncompartmental analysis using PK solver. PK parameters assessed included maximum concentration $\left(C_{\max }\right)$, area under the curve from time 0 to $\infty$ ( $\left(\mathrm{UCC}_{0 \text {-inf }}\right)$, apparent volume of distribution $\left(\mathrm{V}_{\mathrm{d}} / \mathrm{F}\right)$, and apparent clearance $(\mathrm{Cl} / \mathrm{F})$. $\mathrm{AUC}_{0 \text {-inf }}$ represents the total amount of drug available after administration. The $\mathrm{V}_{\mathrm{d}} / \mathrm{F}$ and $\mathrm{Cl} / \mathrm{F}$ parameters illustrate the distribution of drug into body tissues and rate of elimination of drug, respectively. A linear regression was performed to determine how well maternal acetaminophen levels predicted fetal levels.

\section{Results}

In this study, 34 subjects were enrolled at a median age of 32 years (range, 25-39 years). Subjects were primarily Caucasian (31/34 or $91 \%)$, with the remainder Asian (2/34 or $6 \%$ ) or Hispanic (1/34 or $3 \%$ ). The median maternal weight was $82 \mathrm{~kg}$ (range, 62-100 kg). Two women delivered at 38 weeks', 30 delivered at 39 weeks', and 2 women delivered at 40 weeks' gestation. The median newborn birth weight was 3,590 g (interquartile range, 3,403-3,848 g). Noncompartmental PK analysis described acetaminophen disposition in the maternal compartment with a half-life of 84 minutes and $\mathrm{Cl} / \mathrm{F}$ of $28.8 \mathrm{~L} / \mathrm{h}$. In the fetal compartment, acetaminophen half-life was 82 minutes with a $\mathrm{Cl} / \mathrm{F}$ of $31.2 \mathrm{~L} / \mathrm{h}$. Peak concentrations of acetaminophen (maternal $12.3 \mu \mathrm{g} / \mathrm{mL}$; fetal $11.2 \mu \mathrm{g} / \mathrm{mL}$ ) and $\mathrm{V}_{\mathrm{d}} / \mathrm{F}$ (maternal $57.5 \mathrm{~L}$; fetal $61.2 \mathrm{~L}$ ) were similar. Linear regression analysis revealed that maternal acetaminophen level was a significant predictor of fetal level $(p>0.0001)$ and that maternal and fetal levels were very highly correlated $(r=0.91)$. PK parameters and curves are provided in - Fig. $\mathbf{1}$.

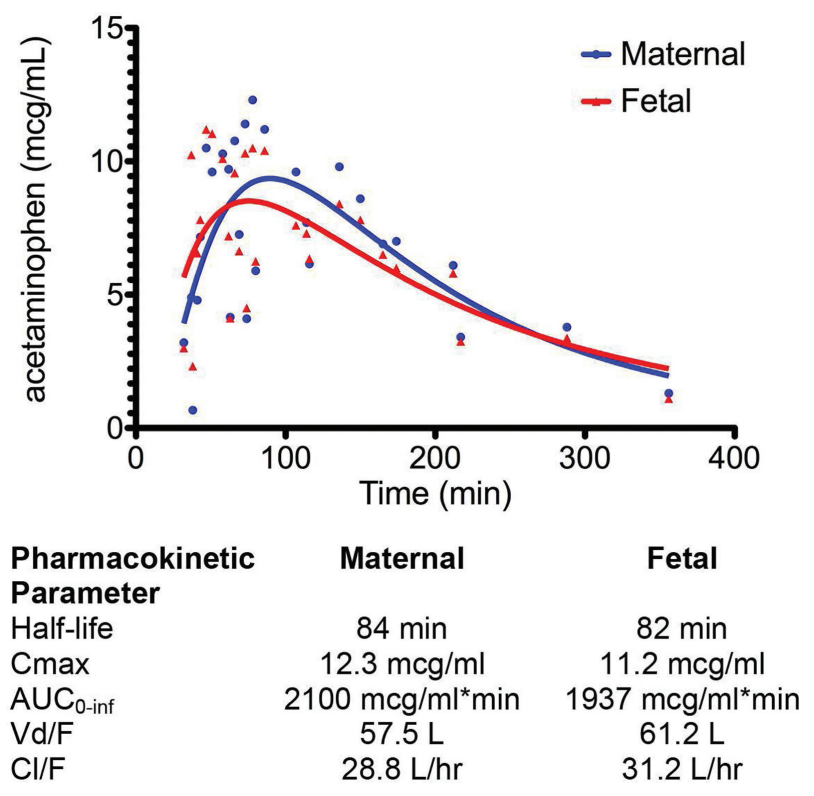

Fig. 1 Acetaminophen PKs in maternal and fetal compartments. Maternal and fetal PK curves are juxtaposed with key parameters listed later. Points on the graph are individual acetaminophen levels with a line of best fit representing the PK curve for the study population. $\mathrm{AUC}_{0 \text {-inf, }}$ area under the curve (time $\left.0-\infty\right)$; $\mathrm{C}_{\text {max }}$, maximum concentration; $\mathrm{Cl} / \mathrm{F}$, apparent clearance; $\mathrm{PK}$, pharmacokinetic; $\mathrm{V}_{\mathrm{d}} / \mathrm{F}$, apparent volume of distribution. 


\section{Conclusion}

Acetaminophen PKs in maternal samples are consistent with values previously reported in pregnant women $(\mathrm{Vd} / \mathrm{F}, 57.5 \mathrm{~L}$; $\mathrm{Cl} / \mathrm{F}, 28.8 \mathrm{~L} / \mathrm{h}$ ), except for a shorter half-life (84 vs. 114 minutes). ${ }^{15}$ They are also consistent with those described in healthy male volunteers in whom serum levels peak between 7.7 and $17.6 \mu \mathrm{g} / \mathrm{mL}^{12}$ and have a half-life of 1.5 to 2.5 hours. ${ }^{16}$ These values are similar to the peak concentration of $12.3 \mu \mathrm{g} / \mathrm{mL}$ and a half-life of 84 minutes seen in our PK model. Maternal and fetal acetaminophen levels are comparable as early as 30 minutes after administration, indicating rapid placental transfer of the drug. Notably, acetaminophen PKs in fetal samples closely parallels behavior of the drug in the maternal system. The time to peak concentration and half-life are similar, and the fetal AUC is nearly the same as maternal AUC (> 90\%). The comparable concentrations of acetaminophen in the maternal and fetal compartments suggest that placental transfer is influenced primarily by rate of blood flow ("flow limited") and not by transporters in the placenta ("diffusion limited").

Fetal acetaminophen exposure has been associated with ADHD, ${ }^{2}$ DILI, ${ }^{3}$ and childhood asthma, ${ }^{4}$ though the threshold for these effects in the fetus is not known. An understanding of placental transfer kinetics is necessary to correlate maternal drug intake to fetal exposure and clinical outcomes. In this study, we have demonstrated that acetaminophen levels in the fetal compartment are nearly identical to maternal levels. The very high correlation between maternal and fetal levels suggests that maternal acetaminophen levels can be used as a surrogate for the extent of fetal exposure.

The modest sample size and lack of information on acetaminophen metabolites are the main weaknesses of the study. Although our findings do provide novel information on the PKs of acetaminophen in pregnant women and their fetuses, acetaminophen levels alone cannot provide precise estimations of fetal risk, as acetaminophen-mediated injury occurs when a toxic metabolite, $\mathrm{N}$-acetyl-p-benzoquinone imine (NAPQI), is formed. Saturation of the metabolic enzymes that inactivate acetaminophen leads to accumulation of NAPQI and subsequent adverse effects. Thus, future studies should assess NAPQI along with acetaminophen levels and utilize a larger sample size to permit a formal population PK analysis. In addition, our observation that transplacental passage of acetaminophen is "flow limited" is based on features of the maternal and fetal PK curves. Confirmation that acetaminophen does not interact with placental transporters may be obtained through ex vivo placental perfusion studies.
Although the current study suggests that fetal exposure to acetaminophen can be predicted using maternal drug levels, further study of acetaminophen metabolism to NAPQI in the fetus is clearly needed to better understand the risk of fetal harm after maternal acetaminophen use. However, our findings suggest that maternal use of acetaminophen at the currently recommended dose is unlikely to lead to accumulation of potentially toxic levels in the fetus.

\section{References}

1 Werler MM, Mitchell AA, Hernandez-Diaz S, Honein MA. Use of over-the-counter medications during pregnancy. Am J Obstet Gynecol 2005;193(3 Pt 1):771-777

2 Liew Z, Ritz B, Rebordosa C, Lee PC, Olsen J. Acetaminophen use during pregnancy, behavioral problems, and hyperkinetic disorders. JAMA Pediatr 2014;168(4):313-320

3 Haibach H, Akhter JE, Muscato MS, Cary PL, Hoffmann MF. Acetaminophen overdose with fetal demise. Am J Clin Pathol 1984; 82(2):240-242

4 Eyers S, Weatherall M, Jefferies S, Beasley R. Paracetamol in pregnancy and the risk of wheezing in offspring: a systematic review and meta-analysis. Clin Exp Allergy 2011;41(4):482-489

5 Wang PH, Yang MJ, Lee WL, Chao HT, Yang ML, Hung JH. Acetaminophen poisoning in late pregnancy. A case report. J Reprod Med 1997;42(6):367-371

6 Levy G, Garrettson LK, Soda DM. Letter: evidence of placental transfer of acetaminophen. Pediatrics 1975;55(6):895-895

7 Byer AJ, Traylor TR, Semmer JR. Acetaminophen overdose in the third trimester of pregnancy. JAMA 1982;247(22):3114-3115

8 Ludmir J, Main DM, Landon MB, Gabbe SG. Maternal acetaminophen overdose at 15 weeks of gestation. Obstet Gynecol 1986; 67(5):750-751

9 Rosevear SK, Hope PL. Favourable neonatal outcome following maternal paracetamol overdose and severe fetal distress. Case report. Br J Obstet Gynaecol 1989;96(4):491-493

10 Stokes IM. Paracetamol overdose in the second trimester of pregnancy. Case report. Br J Obstet Gynaecol 1984;91(3):286-288

11 Briggs G, Freeman R, Yaffe S, eds. Drugs in Pregnancy and Lactation, 6 ed. Philadelphia: Lippincott Williams \& Wilkins; 2002

12 Tylenol professional product information. In: McNeil Consumer Healthcare; 2010

13 Rayburn W, Wible-Kant J, Bledsoe P. Changing trends in drug use during pregnancy. J Reprod Med 1982;27(9):569-575

14 Naga Rani MA, Joseph T, Narayanan R. Placental transfer of paracetamol. J Indian Med Assoc 1989;87(8):182-183

15 Kulo A, van Calsteren K, Verbesselt R, et al. The impact of caesarean delivery on paracetamol and ketorolac pharmacokinetics: a paired analysis. J Biomed Biotechnol 2012;2012:437639. Doi: 10.1155/ 2012/437639

16 Mazaleuskaya LL, Sangkuhl K, Thorn CF, FitzGerald GA, Altman RB, Klein TE. PharmGKB summary: pathways of acetaminophen metabolism at the therapeutic versus toxic doses. Pharmacogenet Genomics 2015;25(8):416-426 